\title{
Finite-bandwidth calculations for charge carrier mobility in organic crystals
}

\author{
V.M. Kenkre \\ Department of Physics and Astronomy, Center for Advanced Studies, University of New Mexico, Albuquerque, NM 87131, USA \\ Received 4 August 2002; accepted 31 October 2002 \\ Communicated by A.R. Bishop
}

\begin{abstract}
Finite-bandwidth effects on the temperature dependence of the mobility of injected carriers in pure organic crystals are explored for a simplifed case of impurity scattering. Temperature-dependent bandwidth effects are discussed briefly through a simplified combination of band and polaronic concepts.
\end{abstract}

(C) 2002 Elsevier Science B.V. All rights reserved.

Keywords: Mobility; Band; Polaron; Organic

\section{Introduction}

In studying charge carrier (electron or hole) transport in organic crystals, one avoids the use of standard band theoretic approaches [1] since the carrier bandwidth is not an overwhelmingly large energy in organic, in contrast to inorganic, materials [2]. Mean free paths calculated on the basis of bare band-theoretic descriptions have been explicitly found to be smaller than a lattice constant [3]. Such findings have necessitated polaronic or hopping theories of transport in organic materials $[4,5]$. The question arises whether this trend $[6,7]$ away from bare-band theory, which has continued in the literature on organic materials in recent times [8], needs to be revised or at least modified in light of recent observations [9] and calculations [10] on pentacene. The large low-temperature mobil-

E-mail address: kenkre@unm.edu (V.M. Kenkre). ity in these materials (orders of magnitude larger than values reported earlier [11] at other temperatures and in other organics), sharp power-law dependence of the mobility on temperature in a wide range $[9,12]$, and nonlinear saturation phenomena [13] which have been interpreted [9] in terms of large bandwidths, all could point to the need for bare-band theory to be considered seriously in organic materials. On the other hand, the bandwidths are believed $[9,10]$ not to be of the order of several eV's as in inorganic materials but smaller. One concludes, therefore, that required is a description valid for intermediate bandwidths $(B)$. To begin the construction of such a description is the main purpose of the present Letter.

Temperature-dependent (Huang-Rhys) $B$ 's arise in polaronic transport $[4,5,14]$. In the past, effective bare $B$ 's, which undergo reduction by polaronic effects in the presence of strong interactions with vibrations, were themselves believed (or shown) to be rather small. With naphthalene as an example, $B \sim 10 \mathrm{meV}$, 
the polaronically reduced $B$ is about a tenth of this value in light of the magnitude of the coupling constants and is thus well surpassed by the thermal energy $k_{B} T$. The latter exceeds $2.5 \mathrm{meV}$ corresponding to $30 \mathrm{~K}$, the lowest temperature in the naphthalene observations [11]. Therefore, although past explanations of mobility observations have certainly made use of the temperature dependence of polaronic bandwidths [4, 5] to address decreasing mobility with increasing temperature [5], polaronic calculations have served as the investigational procedure. Band considerations have either not been used at all [5] or assigned a less important role [4]. Estimates in pentacene raise the possibility that $k_{B} T$ is smaller than $B$ for a part of the observations. To show the results of a simple combination capable of blending finite-band theory and polaronic concepts, is a secondary purpose of the present Letter.

\section{Constant scattering rates and truncated parabolic bands}

Band-theoretic descriptions in inorganic materials effectively take the bandwidth $B$ to be infinite. The well-known textbook formula for the mobility

$\mu=\left(\frac{q}{k_{B} T}\right) \frac{\int_{0}^{\infty} d \varepsilon v^{2}(\varepsilon) \tau(\varepsilon) \rho(\varepsilon) e^{-\varepsilon / k_{B} T}}{\int_{0}^{\infty} d \varepsilon \rho(\varepsilon) e^{-\varepsilon / k_{B} T}}$,

where $k_{B}$ is the Boltzmann constant, and $q, \varepsilon, T, v, \rho$, $\tau$ are the carrier charge, energy, temperature, velocity, density of states, and relaxation time, respectively, has $\infty$ as the upper limit of the energy integrations. We will explore, instead, the consequences of replacing it by the finite value of $B$, suitably modifying the density of states. We consider a one-band model, which means merely that the band gap is taken to be large enough to make interband transitions unimportant, and assume a simple $\rho(\varepsilon)$ having the free-electronic form within the band and to vanish outside:

$\rho(\varepsilon)=N\left(\frac{3 \sqrt{\varepsilon}}{2 B \sqrt{B}}\right)[\theta(\varepsilon)-\theta(\varepsilon-B)]$,

where $\theta$ is the Heaviside step function and $N$ is the number of sites. It is easy to show that the carrier velocity $v(\varepsilon)$ is

$v=\frac{1}{\hbar} \frac{d \varepsilon}{d k}=C \sqrt{B \varepsilon}$.
The constant $C$ equals $(2 / \sqrt{3})(a / \hbar)\left(3 \pi^{2}\right)^{-1 / 3}$ if the system is isotropic in three dimensions, $a$ being the lattice constant, given that the charge carriers are Fermionic ( 2 spin values per orbital state). The thermal distribution in (1) is classical for the standard reasons that the carriers being injected are so small in number, e.g., in time-of-flight observations, that the Fermi distribution is well approximated by a Maxwell distribution. Although space-charge limited conditions of measurement are common now for some experimental setups such as in field-effect-transistor geometry, we will assume here that this assumption is valid. If the need arises, it can always be relaxed in subsequent calculations.

Our present purpose being to get at the simplest consequences of finite $B$ as economically as possible, for most of the calculations here, we will first take the relaxation time $\tau$ to have no energy dependence. Such a case appears in an early analysis of Erginsoy [15]. Whether the entire set of assumptions behind Erginsoy's analysis applies to organic crystals is of no importance in the present context. Our interest lies only in exploring, in the first instance, the consequences of a constant $\tau$ in a finite band system. The finite-band generalization of (1) is then

$$
\begin{aligned}
\mu^{\prime} & =\frac{\mu}{\hbar q C^{2}}=\left(\frac{B}{S}\right) \frac{\int_{0}^{B / k_{B} T} d x x \sqrt{x} e^{-x}}{\int_{0}^{B / k_{B} T} d x \sqrt{x} e^{-x}} \\
& =\left(\frac{B}{S}\right)\left(\frac{B}{k_{B} T}\right) \frac{\int_{0}^{1} d x x \sqrt{x} e^{-x B / k_{B} T}}{\int_{0}^{1} d x \sqrt{x} e^{-x B / k_{B} T}} .
\end{aligned}
$$

Displayed in (4), is the dimensionless ratio $\mu^{\prime}$ of the mobility to $\hbar q C^{2}=\left(4 a^{2} q / 3 \hbar\right)\left(3 \pi^{2}\right)^{-2 / 3}$, and $S$ is the 'scattering energy' $\hbar / \tau$. The dimensionless mobility $\mu^{\prime}$ has been expressed in terms of the dimensionless ratios of the bandwidth to the scattering energy and to the thermal energy: $B / S$ and $B / k_{B} T$. While both expressions in (4) are exact, they can be used respectively in opposite limits most conveniently:

$$
\begin{array}{ll}
\mu^{\prime}=(3 / 2)(B / S) & \text { for } B / k_{B} T \rightarrow \infty, \\
\mu^{\prime}=(3 / 5)(B / S)\left(B / k_{B} T\right) & \text { for } B / k_{B} T \rightarrow 0 .
\end{array}
$$

This $B$ to $B^{2}$ transition as one goes from large bands to small bands arises from the occupation of states in the band. The mobility, which involves the thermal average of the square of the velocity, is proportional to $B k_{B} T$, the product of $B$ and the thermal energy 
in the large bandwidth case, but to $B^{2}$ in the small bandwidth case, because the temperature is so large with respect to $B / k_{B}$ that it makes no contribution: all states in the band are occupied equally. The transition takes on different character if the relaxation time $\tau$ is energy-dependent, the limiting exponents of $B$ being different from 1 and 2, respectively.

The two different ways of scaling the energy apparent in (4) are particular cases $Q=k_{B} T$ and $Q=B$ of scaling with an arbitrary quantity $Q$ having the dimensions of energy:

$\mu^{\prime}=\left(\frac{B}{S}\right)\left(\frac{Q}{k_{B} T}\right) \frac{\int_{0}^{B / Q} d x x \sqrt{x} e^{-x Q / k_{B} T}}{\int_{0}^{B / Q} d x \sqrt{x} e^{-x Q / k_{B} T}}$.

This general expression can be used in additional ways such as by taking $Q=S$ :

$\mu^{\prime}=\left(\frac{B}{k_{B} T}\right) \frac{\int_{0}^{B / S} d x x \sqrt{x} e^{-x S / k_{B} T}}{\int_{0}^{B / S} d x \sqrt{x} e^{-x S / k_{B} T}}$.

In that case, extreme limits of $B / S$ can be studiedif it is very large, which represents the large mean free path case, $\mu^{\prime}$ is proportional to a ratio of Laplace transforms of powers, with $S / k_{B} T$ as the Laplace variable.

The three representations of the mobility given above are useful in understanding limiting behavior of carrier transport in extremes of the three respective ratios of $B, k_{B} T$, and $S$. Generally, one can identify the integrals in (4) with incomplete gamma functions $\gamma$ defined via $\gamma(b, x)=\int_{0}^{x} e^{-t} t^{b-1} d t$, and write, exactly, i.e., for arbitrary relative values of $B, k_{B} T$, and $S$,

$\mu^{\prime}=\left(\frac{B}{S}\right) \frac{\gamma\left(5 / 2, B / k_{B} T\right)}{\gamma\left(3 / 2, B / k_{B} T\right)}$

which can easily be shown to lead to the various respective limits, or rewritten in other ways such as in terms only of error functions and exponentials through the use of the chain condition $\gamma(b+$ $1, x)=b \gamma(b, x)-x^{b} e^{-x}$ and the relation $\gamma(1 / 2, x)=$ $\sqrt{\pi} \operatorname{erf}(\sqrt{x})$.

\section{Power laws}

The sharpness of the power laws in the temperature dependence of the observed $\mu$ in organic crystals, whether in the recent experiments on pentacene [9] or in experiments on other crystals such as naphthalene reported a couple of decades back [12], is impressive. Although Giuggioli et al. [16] have shown recently that visually acceptable fits to the data can be produced by a band theory addressing the partial range of pentacene data from $20-400 \mathrm{~K}$ by combining acoustic and phonon scattering, it is interesting to ask if a true power law form can be obtained as an analytic limit from the expressions. The following simplified approach produces such as a limit. Assume that the mechanism of scattering is such that the relaxation time $\tau$ depends both on the carrier energy $\varepsilon$ and the temperature $T$ as $\tau=\tau_{0}\left(\varepsilon / \varepsilon_{0}\right)^{-p}\left(T / T_{0}\right)^{-r}$ where $\varepsilon_{0}, \tau_{0}$, and $T_{0}$ are a characteristic energy, time, and temperature, respectively. Such expressions arise naturally in many contexts. Thus, $p=1 / 2$ in many semiconductors [17], and $r=1$ for acoustic phonon scattering at high temperatures. Eq. (1) then yields

$$
\begin{aligned}
\mu^{\prime}= & \left(\frac{B \tau_{0}}{\hbar}\right)\left(\frac{\varepsilon_{0}}{k_{B} T_{0}}\right)^{p} \frac{\gamma\left(5 / 2-p, B / k_{B} T\right)}{\gamma\left(3 / 2, B / k_{B} T\right)} \\
& \times\left(\frac{T}{T_{0}}\right)^{-(p+r)} .
\end{aligned}
$$

Whether or not this appears as a power form depends on the particular $T$ dependence of the ratio of the $\gamma$-functions. For $p=0$, one recovers (8) except for the explicit $T^{-r}$ factor. For $p=1$, the $\gamma$-functions cancel and the mobility is seen to display a clear power law $T^{-(1+r)}$. In such a case the value $r=1.7$ could conceivably correspond to the observed $T^{-2.7}$ dependence in pentacene [9]. It is easy to see that, even if the value of $p$ is not 1 , a sharp power law may indeed be the direct apparent consequence of the assumed $\tau$ in an appropriate (although limited) range of temperature, provided the ratio of the $\gamma$-functions in (9) is largely $T$-independent in that range.

\section{Huang-Rhys bandwidths and polaronic expressions}

If polaronic effects are present in carrier transport, it could be argued [9] that some of the physics might be captured by invoking the well-known Huang-Rhys dependence

$B=\widetilde{B}=B_{0} e^{-G^{2} \operatorname{coth}\left(\hbar \Omega / 2 k_{B} T\right)}$, 
where $B_{0}$ is the bare bandwidth, and its exponential reduction occurs as a result of strong interaction via the coupling constant $G$ with vibrations of frequency $\Omega$. For instance, the substitution of (10) in the simple constant- $\tau$ version (8) would yield the normalized mobility

$$
\frac{\mu(T)}{\mu(0)}=\frac{e^{-G^{2}\left[\operatorname{coth}\left(\frac{h \Omega}{2 k_{B} T}\right)-1\right]}}{3 / 2}\left[\frac{\gamma\left(\frac{5}{2}, \frac{\widetilde{B}}{k_{B} T}\right)}{\gamma\left(\frac{3}{2}, \frac{\widetilde{B}}{k_{B} T}\right)}\right] .
$$

Eq. (11) displays the qualitative trend of the low temperature data, but not of the high temperature data, in the acenes. Furthermore, there is always a large enough temperature at which the Huang-Rhys factors reduce the polaron bandwidth enough to make the mean free path smaller than a lattice constant. This forces the band calculation to lose its accuracy and, indeed, its applicability. Crystal momentum becomes an inadequate quantum number in such a case, and a hopping description becomes necessary. In particular, a bridging of the present finite-band calculations and of polaronic theories developed earlier [4,5] is required. How would one carry out such a combination? This important and difficult question, along with application to pentacene observations, is being addressed in our ongoing work. Here we sketch a simplified answer to the question.

Polaronic mobility theory for narrow bands has been successfully applied [5] more than a decade ago to naphthalene observations [11] on the basis of an expression, which, in one of its most simplified forms, may be written as

$\mu=\frac{c q}{k_{B} T}\left(\frac{\widetilde{B} a}{\hbar}\right)^{2}\left(\frac{1}{\alpha}\right) I_{0}\left(\frac{2 G^{2}}{\sinh \left(\frac{\hbar \Omega}{2 k_{B} T}\right)}\right)$,

where $c$ is a numerical constant and $I_{0}$ is a modified Bessel function. The scattering rate $\alpha$ is essentially the constant $1 / \tau$ appearing in band expressions shown earlier in this Letter. The expression comes about from the time integration of the velocity autocorrelation function. The velocity autocorrelation function is a product of a polaron part which arises from the interaction of the charge carrier with vibrations and a part $e^{-\alpha t}$ which arises from interaction with static defects [18]. Eq. (12) is appropriate to a narrow band. An extension of the narrow-band expression to an intermediate-band situation may be made by using the calculation given earlier in this Letter to obtain the static part $e^{-\alpha t}$. It leads to the replacement of $\alpha$ in (12) by an effective $\alpha$ which is dependent on $T$ and $B$ in addition to being proportional to the constant $1 / \tau$. The result is (with $c^{\prime}$ a numerical constant)

$$
\mu=c^{\prime} q \tau\left(\frac{a}{\hbar}\right)^{2} \widetilde{B}\left[\frac{\gamma\left(\frac{5}{2}, \frac{\widetilde{B}}{k_{B} T}\right)}{\gamma\left(\frac{3}{2}, \frac{\widetilde{B}}{k_{B} T}\right)}\right] I_{0}\left(\frac{2 G^{2}}{\sinh \left(\frac{\hbar \Omega}{2 k_{B} T}\right)}\right) .
$$

The general result (13) combines the polaronic and band-theoretic character in a simple way. It reduces to the polaronic form (12) for large $T$, i.e., for $k_{B} T \gg \widetilde{B}$ where $\widetilde{B}$ is the reduced bandwidth given by (10). In this limit, the reduced band is fully occupied and the ratio of the two $\gamma$-functions simplifies to $(3 / 5)\left(\widetilde{B} / k_{B} T\right)$. In the opposite limit of small temperatures, $k_{B} T \ll \widetilde{B}$, the occupation of the band is controlled by the temperature, the zero- $T$ limit of the ratio of the $\gamma$-functions is simply $3 / 2$, and (13) simplifies to the band result. Indeed, if also $k_{B} T \ll \hbar \Omega / 2$, the argument of the $I$-Bessel function is negligible and the Bessel function is simply 1 . The mobility then reduces to (5) with the zero- $T$ reduced bandwidth $B_{0} e^{-G^{2}}$ in place of $B$ in (5). Generally, the mobility, normalized

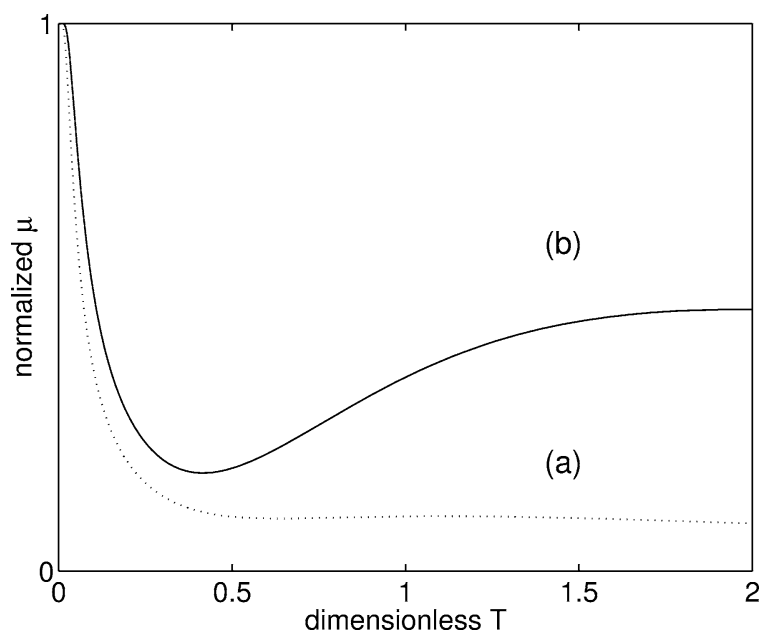

Fig. 1. Dependence of the mobility $\mu$ on the temperature $T$ plotted for two (arbitrary) parameter combinations. In units of $\hbar \Omega$, the bare bandwidth $B_{0}$ is 0.5 and the coupling constant $g$ is 1.5 in (a) while $B_{0}$ is 2 and $g$ is 1.8 in (b). The temperature $T$ is plotted in units of $\hbar \Omega / 2 k_{B}$ and $\mu$ is normalized to its value at $T=0$. Qualitatively only, (a) and (b) have resemblance to naphthalene and pentacene reported data, respectively. 
to its $T=0$ value, is

$\frac{\mu(T)}{\mu(0)}=\mu_{a h}(T) I_{0}\left(\frac{2 G^{2}}{\sinh \left(\frac{\hbar \Omega}{2 k_{B} T}\right)}\right)$,

where $\mu_{a h}(T)$ is the right-hand side of (11).

The ad hoc expression (11) obtained by straightforward substitution of the bare bandwidth $B$ by the polaronic reduced bandwidth $\widetilde{B}$ in (8) differs precisely by the introduction in (14) of the Bessel function factor. This agrees with the well-known fact $[4,5]$ that the excess of that factor over 1 represents the hopping contribution of the mobility. We plot (14) in Fig. 1. Parameters chosen are arbitrary but illustrate the respective behaviors reported in the literature for naphthalene where a flat temperature dependence is observed [11], and for pentacene where a turn-over with increasing mobility in the higher temperature region dependence has been reported [9].

\section{Remarks}

Through simplified calculations, we have presented here arguments towards an intermediate finite-bandwidth theory which extends previous narrow-band treatments. The new results obtained are: finite-band effects stemming from full occupation of the band at temperatures large enough so that the thermal energy exceeds the bandwidth, $B$ to $B^{2}$ transition of the mobility dependence on the bandwidth with variation of temperature, an indication of how power laws might arise as analytical limits of appropriate scattering mechanisms, the specific form of the mobility under the ad hoc Huang-Rhys temperature dependence of the bandwidths and the relation of such an expression to polaronic expressions, and a usable interpolation formula which combines polaronic and bare-band characters.

These calculations should by no means be treated as a complete theory of polaronic and band effects. More realistic analyses of the band transport are being carried out by Giuggioli et al. [16], taking into account acoustic and optical phonon interactions which lead to striking new finite-band effects. While not totally accurate, the simple truncated free-carrier density of states used here has the correct broad (bare) band limit in contrast to Gaussian forms [4] and should, therefore, be able to capture the essential physics at that end. It is hoped that the present simple calculations will contribute towards the construction of a general picture of quasiparticle transport in pure organic crystals which treats both the temperature dependence of the mobility, and high field effects such as velocity saturation that have been treated recently [13].

\section{Acknowledgements}

It is a pleasure to acknowledge conversations with John Andersen, Luca Giuggioli and Paul Parris. This research was supported in part by the NSF under grant DMR-0097204 and by the Los Alamos National Laboratory under a contract to the Consortium of the Americas for Interdisciplinary Science of the University of New Mexico.

\section{References}

[1] J.M. Ziman, Electrons and Phonons, Clarendon, Oxford, 1982.

[2] M. Pope, C.E. Swenberg, Electronic Processes in Organic Crystals and Polymers, New York, Oxford, 1999.

[3] J.D. Andersen, C.B. Duke, V.M. Kenkre, Phys. Rev. Lett. 51 (1983) 2202.

[4] R. Silbey, R.W. Munn, J. Chem. Phys. 72 (1980) 2763.

[5] V.M. Kenkre, J.D. Andersen, D.H. Dunlap, C.B. Duke, Phys. Rev. Lett. 62 (1989) 1165.

[6] L.B. Schein, Philos. Mag. B 65 (1992) 795; L.T. Pautmeier, J.C. Scott, L.B. Schein, Chem. Phys. Lett. 197 (1992) 568.

[7] V.M. Kenkre, D.H. Dunlap, Philos. Mag. B 65 (1992) 831.

[8] P.E. Parris, V.M. Kenkre, D.H. Dunlap, Phys. Rev. Lett. 87 (2001) 126601.

[9] J.H. Schön, Ch. Kloc, B. Batlogg, Phys. Rev. Lett. 86 (2001) 3843 ;

J.H. Schön, Ch. Kloc, B. Batlogg, Phys. Rev. B 63 (2001) 245201.

[10] J.L. Bredas, D. Beljonne, J. Cornil, J.Ph. Calbert, Z. Shuai, R. Silbey, Synth. Met. 125 (2002) 107.

[11] L.B. Schein, C.B. Duke, A.R. McGhie, Phys. Rev. Lett. 40 (1978) 197.

[12] W. Warta, N. Karl, Phys. Rev. B 32 (1985) 1172.

[13] V.M. Kenkre, P.E. Parris, Phys. Rev. B 65 (2002) 205104; V.M. Kenkre, P.E. Parris, Phys. Rev. B 65 (2002) 245106.

[14] T. Hostein, Ann. Phys. (N.Y.) 8 (1959) 340.

[15] C. Erginsoy, Phys. Rev. 79 (1950) 1013.

[16] L. Giuggioli, J.D. Andersen, V.M. Kenkre, preprint.

[17] E.M. Conwell, High Field Transport in Semiconductors, Academic Press, New York, 1967.

[18] V.M. Kenkre, Phys. Lett. A 65 (1978) 391. 